\title{
Probing embedded star clusters in the HII complex NGC 6357 with VVV^
}

\author{
E. F. Lima ${ }^{1,2}$, E. Bica ${ }^{1}$, C. Bonatto ${ }^{1}$, and R. K. Saito ${ }^{3}$ \\ ${ }^{1}$ Universidade Federal do Rio Grande do Sul, Departamento de Astronomia CP 15051, Porto Alegre 91501-970, Brazil \\ e-mail: eliade.lima@ufrgs.br, [bica;charles]@if.ufrgs.br \\ 2 Departamento de Física, CCNE, Universidade Federal de Santa Maria, 97105-900 Santa Maria, RS, Brazil \\ ${ }^{3}$ Departamento de Física, Universidade Federal de Sergipe, Av. Marechal Rondon s/n, 49100-000, São Cristóvão, Brazil
}

Received 14 November 2013 / Accepted 10 June 2014

\section{ABSTRACT}

\begin{abstract}
Context. NGC 6357 is an active star-forming region located in the Sagittarius arm that displays several star clusters, which makes it a very interesting target to investigate star formation and early cluster evolution.

Aims. We explore NGC 6357 with the VISTA Variables in the Vía Láctea (VVV) photometry of seven embedded clusters (ECs), and one open cluster projected in the outskirts of the complex.

Methods. Photometric and structural properties (age, reddening, distance, core, and total radii) of the star clusters were derived. VVV saturated stars were replaced by their 2MASS counterparts. Field-decontaminated VVV photometry was used to analyse colourmagnitude diagrams (CMDs) and stellar radial density profiles and determine astrophysical parameters.

Results. We report the discovery of four ECs and one intermediate-age cluster in the complex area. We derive a revised distance estimate for NGC 6357 of $1.78 \pm 0.1 \mathrm{kpc}$ based on the cluster CMD morphologies. Of the ECs, one contains the binary star WR 93, while the remaining ones are dominated by pre-main sequence stars, young stellar objects, and/or and have a developed main sequence. These features reflect a significant age spread among the clusters. Evidence is found that the relatively populous cluster Pismis 24 hosts two subclusters.
\end{abstract}

Key words. open clusters and associations: general - open clusters and associations: individual: NGC 6357 instrumentation: adaptive optics

\section{Introduction}

Star-forming complexes are, in general, major building blocks of the large-scale structure of galaxies and important sites to study how massive stars form (Russeil et al. 2010). In particular, Galactic embedded and open clusters are excellent probes of the structure and evolution of the disk and spiral arms (Carvalho et al. 2008; Lada \& Lada 2003; Friel 1995). Embedded clusters (ECs) can be partially or fully immersed in embryonic molecular clouds and HII regions. According to Leisawitz et al. (1989), all clusters younger than $\sim 5 \mathrm{Myr}$ are connected at least to one large molecular cloud or HII region.

Recently, our group studied the stellar content of the Sh2-132 HII region, a star-forming complex hosting at least four ECs and presenting evidence of triggered star formation and hierarchical structuring (Saurin et al. 2010). Sequential star formation in giant molecular clouds was also studied by Camargo et al. (2011). Observationally, low-mass star clusters younger than about $10 \mathrm{Myr}$ present an underpopulated, developing main sequence (MS) and a more populous feature of premain sequence (PMS) stars (Bonatto \& Bica 2009a). Studies of very young star clusters hosting PMS and MS stars have produced well-defined colour-magnitude diagrams (CMDs), radial density profiles (RDPs), and mass functions (Bonatto \& Bica 2009c). Recently, additional tools were developed by our group, allowing us to obtain reliable fundamental parameters of early

* Based on observations taken with the ESO VISTA Public Survey VVV, Programme ID 179.B-2002 and data from the 2MASS VizieR Catalog II/246. cluster phases (Bonatto et al. 2012a,b). The present paper focuses on the ECs in the NGC 6357 complex.

NGC 6357 ( $\equiv \mathrm{W} 22 \equiv \mathrm{RCW} 131 \equiv \mathrm{Sh} 2-11)$ is a large HII region complex that consists of a shell of about $60 \times$ $40 \operatorname{arcmin}^{2}$, bright optical nebulosities in different evolutionary stages, OB stars belonging to the populous open cluster Pismis 24, and young stellar object (YSO) candidates (Felli et al. 1990; Bohigas et al. 2004; Wang et al. 2007; Russeil et al. 2010; Fang et al. 2012). Persi et al. (1986) showed that the whole HII complex is an active area of recent and on-going star formation. The shell has been interpreted as an ionized gas bubble created by the strong winds of the current massive stars in Pismis 24 or by a previous generation (Lortet et al. 1984; Bohigas et al. 2004; Wang et al. 2007). The total amount of molecular gas related to the large shell was estimated by Cappa et al. (2011) to be $1.4 \times 10^{5} M_{\odot}$.

Early optical studies of NGC 6357 and Pismis 24 revealed 20 O-type and early B-type stars (Moffat \& Vogt 1973; Neckel 1978, 1984; Lortet et al. 1984), including a binary system (HD 157504) composed of a WC7 Wolf-Rayet star (WR 93) and an 07-9 star (van der Hucht 2001). Two of the cluster members, namely Pismis 24-1 (HDE 319718) and Pismis 24-17, were recently classified as spectral type O3.5, some of the brightest and bluest stars known (Massey et al. 2001; Walborn et al. 2002). The total-to-selective extinction ratio $\left(R_{V}\right)$ towards NGC 6357 appears to be about 3.5 (Russeil et al. 2012; Bohigas et al. 2004).

A wide range of distances (1.1-2.6 kpc) has been derived for NGC 6357. This is usually estimated from the distance of Pismis 24. The most recent determination is that of 
Table 1. Star clusters towards NGC 6357.

\begin{tabular}{|c|c|c|c|c|c|c|}
\hline $\begin{array}{l}\text { Cluster } \\
\text { (1) }\end{array}$ & $\begin{array}{c}\alpha \\
(\mathrm{J} 200) \\
(2)\end{array}$ & $\begin{array}{c}\delta \\
(\mathrm{J} 200) \\
(3)\end{array}$ & $\begin{array}{l}l \\
\left({ }^{\circ}\right) \\
(4)\end{array}$ & $\begin{array}{l}b \\
\left({ }^{\circ}\right) \\
(5)\end{array}$ & $\begin{array}{c}R \\
(\operatorname{arcmin}) \\
(6)\end{array}$ & $\begin{array}{c}\text { Comments } \\
\text { (7) }\end{array}$ \\
\hline Pismis $24^{a}$ & $17: 24: 44$ & $-34: 11: 56$ & 353.17 & 0.89 & 1.5 & Optical EC \\
\hline $\operatorname{BDS} 101^{b}$ & $17: 25: 34$ & $-34: 23: 09$ & 353.11 & 0.65 & 0.7 & Infrared EC \\
\hline ESO $392-S C 11^{c}$ & $17: 25: 32$ & $-34: 24: 20$ & 353.09 & 0.64 & 1.7 & EC or OB association? \\
\hline VVV CL164 $^{d}$ & $17: 24: 31$ & $-34: 13: 15$ & 353.12 & 0.92 & 0.5 & Infrared OC \\
\hline VVV CL165 & $17: 24: 45$ & $-34: 11: 28$ & 353.18 & 0.89 & 0.2 & Subcluster of Pismis 24? \\
\hline VVV CL166 ${ }^{d}$ & $17: 24: 47$ & $-34: 12: 36$ & 353.17 & 0.88 & 0.2 & Subcluster of Pismis $24 ?$ \\
\hline VVV CL167 ${ }^{d}$ & 17:25:09 & $-34: 11: 13$ & 353.23 & 0.83 & 0.6 & Infrared EC surrounding WR 93 \\
\hline
\end{tabular}

Notes. Columns 2 to 5: optimised central coordinates. Col. 6: the radii were set by eye for decontamination purposes (Sect. 4.1). ${ }^{(a)}$ Moffat \& Vogt (1973). ${ }^{(b)}$ Bica et al. (2003). ${ }^{(c)}$ Sect. 2. ${ }^{(d)}$ Discovered in the present paper.

Fang et al. (2012), who give $1.7 \pm 0.2 \mathrm{kpc}$. The kinematic distance is $d_{\odot}=1.0 \pm 2.3 \mathrm{kpc}$ (Wilson et al. 1970). Neckel (1978) obtained $d_{\odot}=1.74 \pm 0.31 \mathrm{kpc}$, and Massey et al. (2001) found a distance of $2.56 \pm 0.10 \mathrm{kpc}$ for Pismis 24. Conti \& Vacca (1990) derived for WR $93 d_{\odot}=1.1 \mathrm{kpc}$ and van der Hucht (2001) $d_{\odot}=1.74 \mathrm{kpc}$.

Optical, radio continuum, and near- and mid-IR images of NGC 6357 (Cappa et al. 2011) indicate the giant nature of this complex and its evolved character, which suggest that it is a suitable laboratory for early dynamical and hydrodynamical evolution. The number of ECs in a given star-forming region is fundamental for studying the gas expulsion and dynamical evolution effects (Carvalho et al. 2008). By using the recent VISTA Variables in the Vía Láctea (VVV) $J H K_{\mathrm{S}}$ data (Minniti et al. 2010), we have analyse in detail the stellar clusters detected in NGC 6357. We employ a field-decontamination algorithm (Bonatto \& Bica 2007b) adapted to VVV photometry to analyse CMDs, and RDPs and determine the astrophysical parameters. The present analysis of the ECs shows structural variations as well as morphology diversity of MS and PMS evolutionary sequences in CMDs. Based on this, we infer the star formation history in the complex.

This paper is organised as follows: in Sect. 2 the stellar cluster sample is described. In Sect. 3 we provide details on the VVV data. In Sects. 4 and 5, the cluster photometric and structural analyses are carried out. In Sect. 6 we discuss the results and give conclusions.

\section{Stellar clusters in NGC 6357}

Several studies on NGC 6357 are available in the literature, in particular on the cluster Pismis 24. The WEBDA ${ }^{1}$ database locates the centre of Pismis 24 at $\alpha(2000)=17^{\mathrm{h}} 25^{\mathrm{m}} 32^{\mathrm{s}}$ and $\delta(2000)=-34^{\circ} 25^{\prime} 00^{\prime \prime}$, and provides a distance from the Sun $d_{\odot} \sim 1.99 \mathrm{kpc}$, reddening $E(B-V)=1.72$, and age of $10 \mathrm{Myr}$. WR 93 has been considered a member of Pismis 24 (Massey et al. 2001). However, this star is located $4^{\prime}$ away from the cluster center. We investigate this question in more detail.

To the south-east $\left(\alpha(2000)=17^{\mathrm{h}} 25^{\mathrm{m}} 32^{\mathrm{s}}\right.$ and $\delta(2000)=$ $\left.-34^{\circ} 24^{\prime} 20^{\prime \prime}\right)$ of the complex an object is located that was first reported as ESO392-SC11 (Lauberts 1982). It has also been reported as AH03 J1725-34.4 (Archinal \& Hynes 2003), and BDS 100 (Bica et al. 2003), G351.1+0.7 cluster (Damke et al. 2006), and is also indicated in Moisés et al. (2011). This extended $\left(\sim 3.5^{\prime}\right)$ overdensity appears to be related to the general young population of the complex. It is classified as a star cluster,

\footnotetext{
1 http://www. univie.ac.at/webda/webda.html
}

see for example the DAML02 database ${ }^{2}$ (Dias et al. 2002). The data employed the designation AH03 J1725-34.4 and shows an apparent diameter of $2.6^{\prime}$, a distance of $290 \mathrm{pc}$ (too short for the NGC 6357 complex), and an age of 7 Myr. We here use VVV data to constrain not only parameters, but the nature itself of this object as a young stellar cluster or an OB association (Sect. 4.4). We also focus on the compact cluster BDS 101 (Table 1) found by Bica et al. (2003), which is located at $\sim 1^{\prime} \mathrm{NW}$ of the centre of ESO392-SC11.

We show in Table 1 the star clusters analysed in this work. Four star clusters were discovered by two of us (E. L. and E. B.). They are given VVV cluster identifications, in continuation of the recent cluster numbering by Borissova et al. (2014). They span the interval VVV CL164 to VVV CL167, according to the VVV designations.

In Fig. 1 we show the angular distribution of the studied objects on a digitized sky survey $\left(\mathrm{DSS}^{3}\right)$ image of the nebular complex NGC 6357. We can identify the stellar clusters in shell-like features from Cappa et al. (2011). Pismis 24 (together with VVV CL165 and VVV CL166) is located near the border of G353.12+0.86. VVV CL167 might be related to a ring nebula. Finally, BDS 101 and ESO 392-SC 11 are located in G353.1+0.6.

In Fig. 2 we show a VVV $J H K_{\mathrm{s}}$ zoomed image of three embedded clusters and one open cluster in the sample. We emphasize the difference between embedded and open stellar clusters, the former are embedded in parental molecular clouds and the stellar content is in general dominated by PMS stars (e.g. Lada \& Lada 2003; Bonatto et al. 2006b). On the other hand, OCs are dynamical survivours of the embedded phase (Bastian \& Goodwin 2006). Pismis 24 has very massive stars in the central region, and attached to the north is the bright dust/HII region G353.2+0.9 (Cappa et al. 2011). BDS101 is a compact EC, while the loose EC VVV CL167 is older. VVV CL164 is an OC projected in the NGC 6357 area (Sect. 4.2.4).

\section{VVV photometry and data}

The VVV is an ESO Public Survey scanning the Galactic bulge and plane with the $4 \mathrm{~m}$ class VISTA telescope at Paranal (Minniti et al. 2010). The total observed area is about $562 \mathrm{deg}^{2}$, scanning $-10.0^{\circ} \lesssim b \lesssim+10.5^{\circ}$ and $-10.3^{\circ} \lesssim l \lesssim+5.1^{\circ}$ in the bulge, and within $294.7^{\circ} \lesssim l \lesssim 350.0^{\circ}$ and $-2.25^{\circ} \lesssim b \lesssim+2.25^{\circ}$ in the plane. The VVV survey observes in five passbands, namely $Z(0.87 \mu \mathrm{m}), Y(1.02 \mu \mathrm{m}), J(1.25 \mu \mathrm{m}), H(1.64 \mu \mathrm{m})$, and

\footnotetext{
2 http://www.astro.iag.usp.br/ocdb/file/clusters.txt

3 http://archive.eso.org/dss/dss
} 


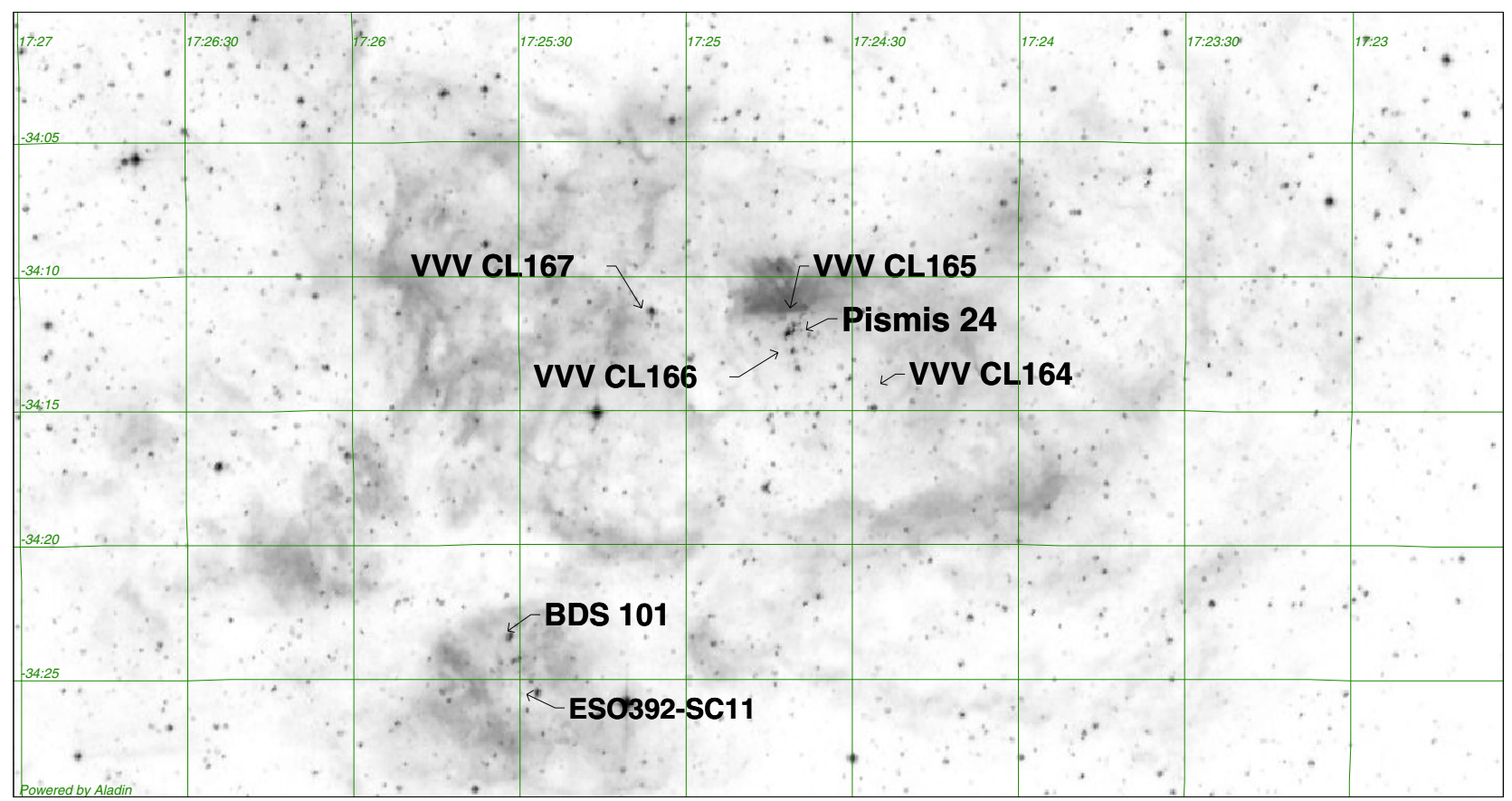

Fig. 1. 55' $\times 30^{\prime}$ DSS R image of the emission nebula NGC 6357. We indicate the present sample of star clusters.
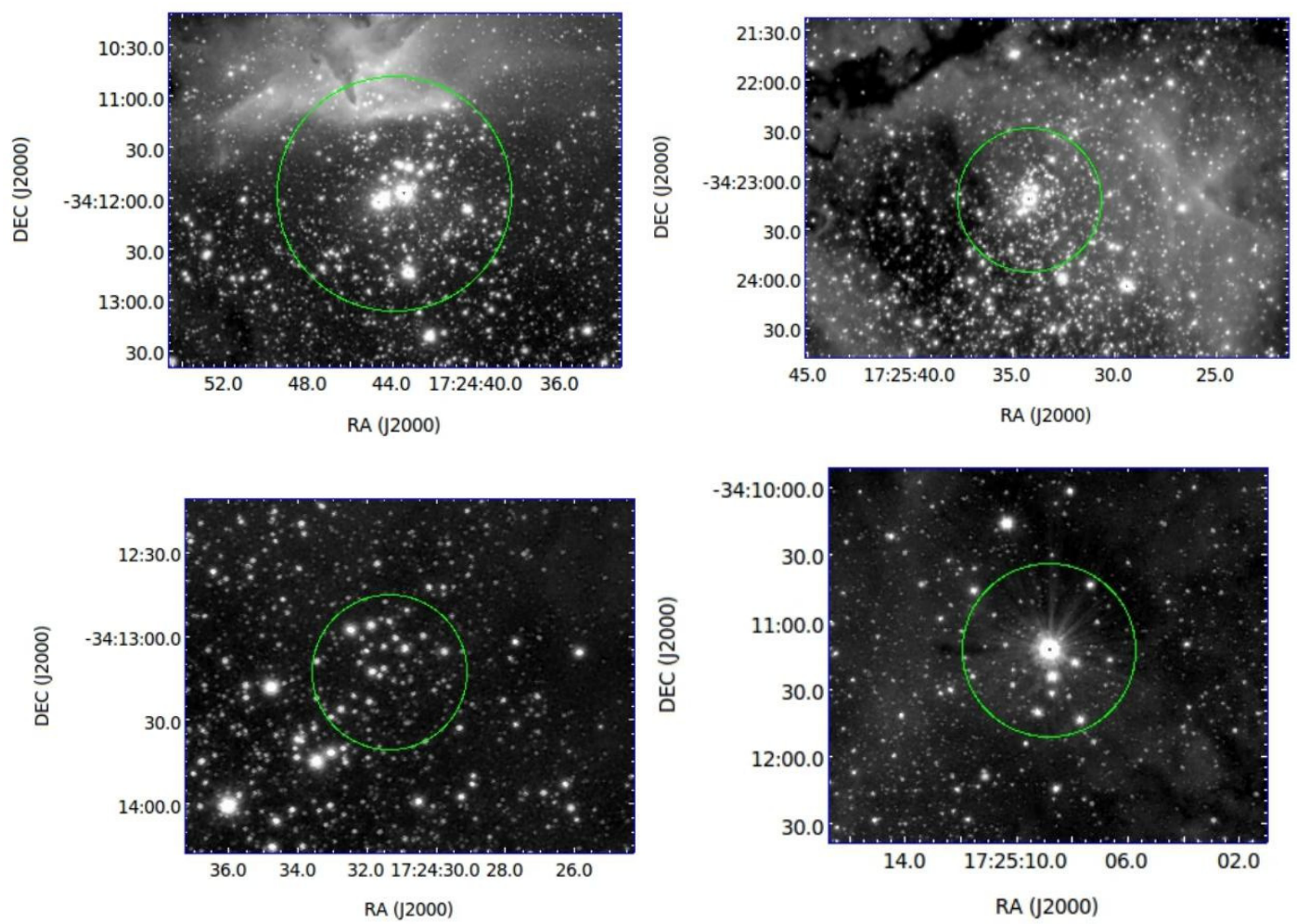

Fig. 2. Combined $J H K_{\mathrm{S}}$ VVV images of clusters in the nebula NGC 6357. Top panels: Pismis 24 (left), BDS 101 (right). Bottom panels: newly found clusters VVV CL164 (left) and VVV CL167 (right). The circles indicate the angular sizes of the clusters (Table 1).

$K_{\mathrm{S}}(2.14 \mu \mathrm{m})$. It also conducts a variability campaign in the $K_{\mathrm{S}}$-band alone, with $\sim 100$ pointings spanning six years $(2010$ 2016). The $J H K_{\mathrm{S}}$ observations were completed during the first semester of 2011. The present work is based on $J, H$ and $K_{\mathrm{S}}$ photometry from VVV data release 1 (DR1; Saito et al. 2012).

Each unit of VISTA observations is called a tile, consisting of six individual pointings (or pawprints) and covers a $1.64 \mathrm{deg}^{2}$ field of view. To fill up the VVV area, a total of 348 tiles are used, with 196 covering the bulge (a $14 \times 14$ grid) and 152 the Galactic plane (a $4 \times 38$ grid). The NGC 6357 complex appears in the VVV bulge/disk tiles b329 and b343.

Photometric catalogues for the VVV images are provided by the Cambridge Astronomical Survey Unit (CASU) ${ }^{4}$. The catalogues contain the positions, magnitudes, and some shape

4 http://casu.ast.cam.ac.uk/vistasp/ 
measurements obtained from different apertures, with a flag indicating the most probable morphological classification. The limiting magnitude for the aperture photometry of the catalogues occurs at $K_{\mathrm{S}}=18 \mathrm{mag}$ in most disk fields. The VVV data are in the natural VISTA Vegamag system, with the photometric calibrations in $J H K_{\mathrm{S}}$ performed using the VISTA magnitudes of unsaturated $2 \mathrm{MASS}^{5}$ stars present in the images. The present work is based on the derived colours and magnitudes established by the CASU v1.3 pipeline reduction. The detailed account of the CASU pipeline can be found in Irwin et al. (2004).

Only sources with VVV $K_{\mathrm{S}}$ photometry defined as stellar were selected (sources with a Gaussian sigma parameter between 0.9 and 2.2). Saturated stars in the VVV data, usually brighter than $J, H$ and $K_{\mathrm{S}} \sim 11 \mathrm{mag}$, were replaced by the respective 2MASS magnitudes.

\section{Star cluster analyses}

In addition to analysing open clusters (e.g. Bonatto \& Bica 2009a), our group has also studied embedded clusters in detail by developing tools for extracting information from the CMDs and RDPs (e.g. Bonatto \& Bica 2009c, 2010).

NGC 6357 in itself is an astrophysical laboratory, not only because of its numerous interstellar structures, such as filaments, bubbles, and knots, but also because of its ECs (Table 1). The VVV photometry provides an adequate means to explore them in this crowded field towards the central disk and bulge.

\subsection{Field-star decontamination}

Field-star decontamination is usually required to identify and characterise star clusters. To distingguish field and cluster stars we used a statistical decontamination algorithm (Bonatto \& Bica 2007a, 2010) adapted to the photometric depth of the VVV. The comparison fields used for the decontamination depend on the projected distribution of individual stars and the presence of other clusters and/or clumpy extinction due to dark clouds in the area. Examples are a ring around the cluster or some other comparison field selected in its vicinity. The algorithm measures the relative number densities of probable field and cluster stars in cubic CMD cells with axes along the $J$ magnitude and $(J-H)$ and $\left(J-K_{\mathrm{S}}\right)$ colours. It (i) divides the range of CMD magnitude and colours into a 3D grid; (ii) estimates the number density of field stars in each cell based on the number of comparison field stars with similar magnitude and colours as those in the cell; and (iii) subtracts the expected number of field stars from each cell. Input algorithm parameters are the cell dimensions $\Delta J=1.0$ and $\Delta(J-H)=\Delta\left(J-K_{\mathrm{S}}\right)=0.2 \mathrm{mag}$. Summing over all cells, each grid setup produces a total number of member stars $\left\langle N_{\text {mem }}\right\rangle$, and by repeating this procedure for the 729 different setups (different cell sizes and their positionings), we obtained the average number of members $\left\langle N_{\text {mem }}\right\rangle$. Each star was ranked according to the number of times it survived all runs (survival frequency), and only the $\left\langle N_{\text {mem }}\right\rangle$ highest ranked stars were accepted as cluster members and transposed to the respective decontaminated CMD.

We decontaminated CMDs to investigate the nature of starcluster candidates and derived their astrophysical parameters. In summary, we applied (i) field-star decontamination to uncover the intrinsic CMD morphology, which is essential to derive reddening, age, and distance to the Sun; and (ii) colour-magnitude

\footnotetext{
5 The Two Micron All Sky Survey, All Sky data release (Skrutskie et al. 2006).
}

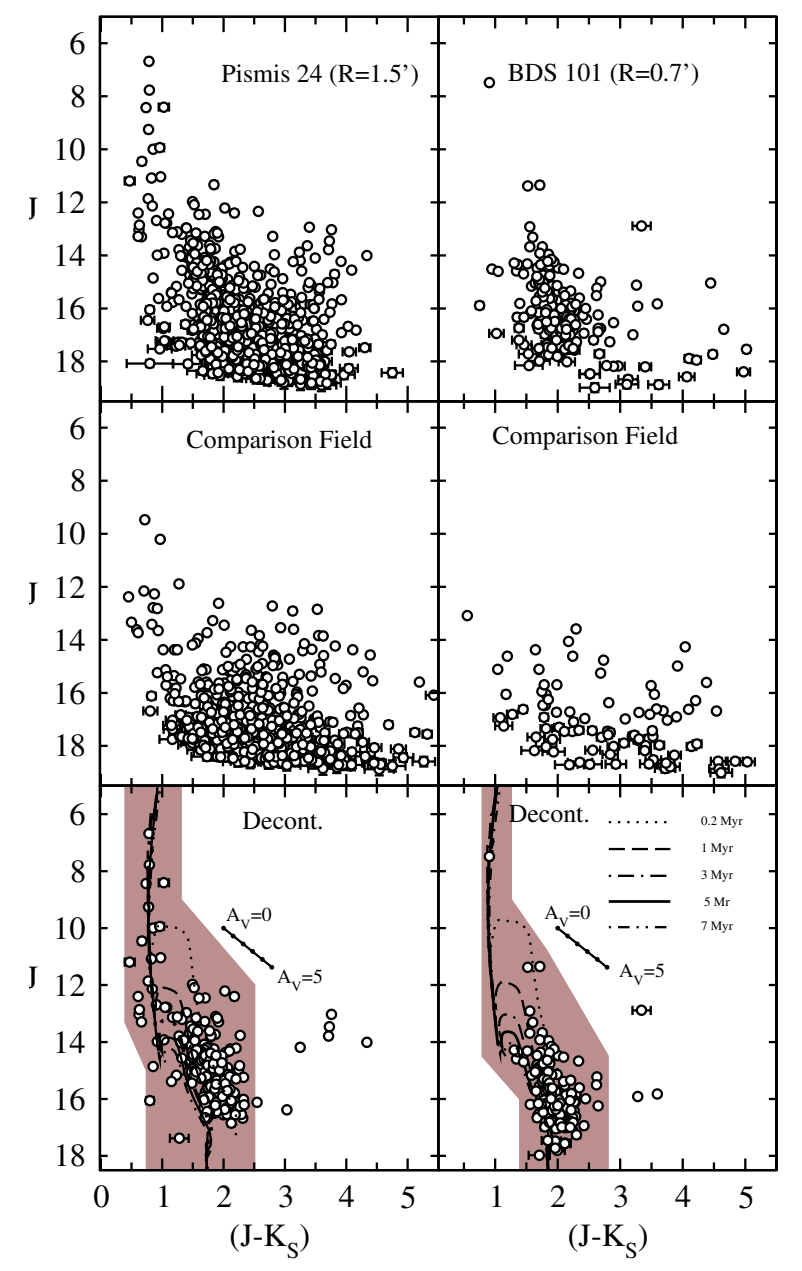

Fig. 3. Top-left panel: $J \times\left(J-K_{\mathrm{S}}\right) \mathrm{CMDs}$ of Pismis 24 showing the observed photometry for representative cluster and field regions. Middleleft panel: equal-area extraction from the comparison field. Bottom-left panel: the decontaminated CMDs fitted with Padova isochrones $0.2,1$, 3,5 , and $7 \mathrm{Myr}$, together with the colour-magnitude filter (brown polygon) used to isolate the MS and PMS stars. Right panels: the same for BDS 101. The reddening vector for $A_{v}=0-5$ mag is shown.

(CM) filters (Fig. 3) to exclude stars different from those of the CMD sequence. The latter filters are wide enough to include cluster MS and PMS stars together with the photometric uncertainties and binary star effects (Sect. 4.2). In the following sections we use this tool several times in the analyses. The latter procedure is required for intrinsic stellar RDPs. In particular, the use of field-star decontamination in constructing CMDs has proved to constrain age and distance much more effectivelly than the raw (observed) photometry (e.g. Bonatto \& Bica 2010).

\subsection{Colour-magnitude diagrams}

The CMDs built with the raw photometry of the present objects are shown in the top panels of Figs. 3, 4, 6 and 11. For qualitative comparison, CMDs extracted from equal-area comparison fields are shown in the middle panels. The decontamination itself was based on as large as possible field areas. Finally, the decontaminated CMDs are shown in the bottom panels, together with the respective CM filters.

Fundamental parameters (Table 2) were derived by means of the constraints provided by the field-decontaminated CMD morphologies that combine the MS and PMS distributions. 


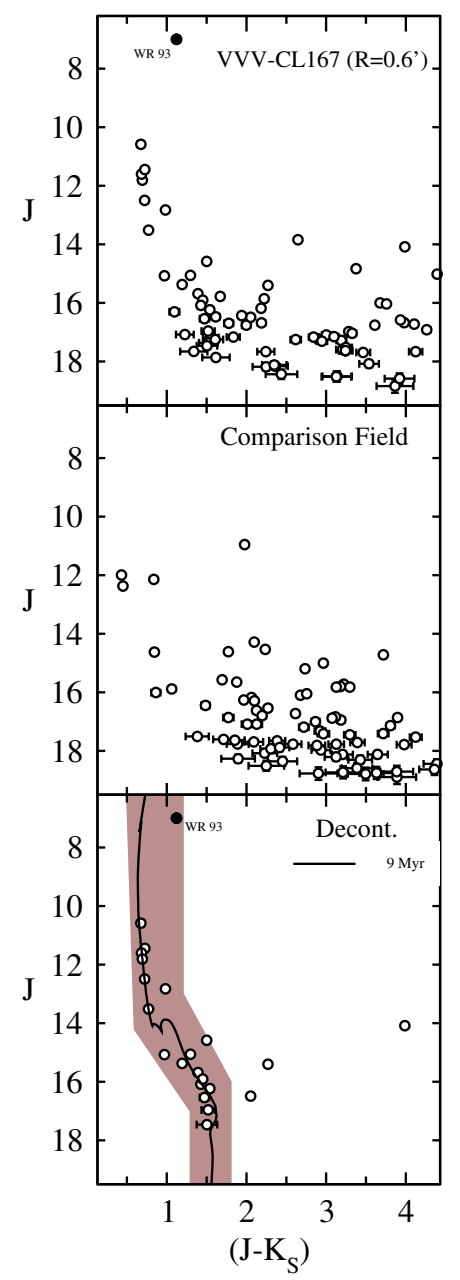

Fig. 4. Top panel: observed $J \times\left(J-K_{\mathrm{S}}\right) \mathrm{CMD}$ of the region $R<0.6^{\prime}$ of VVV CL167. Middle panel: equal-area extraction from the comparison field. Bottom panel: the decontaminated CMD best-fit Padova isochrone is $9 \mathrm{Myr}$ (solid line) together with the CM filter (brown polygon). WR 93 is indicated.

Historically, different approaches have been used to extract astrophysical parameters from isochrone fits. A review of these methods is given by Naylor \& Jeffries (2006). In the present cases fits were matched by eye, taking the combined MS and PMS stellar distribution as constraint. Throughout the paper we use the Padova isochrones with solar metallicity $(Z=0.0019)^{6}$ (Bressan et al. 2012) computed for the VISTA Z, Y, J, H, and $K_{\mathrm{S}}$ filters. The derived fundamental parameters are given in Table 2, where we also provide the Galactocentric distance $\left(R_{\mathrm{GC}}\right)$, which is based on the derived value of the Sun's distance to the Galactic centre $R_{\odot}=7.2 \mathrm{kpc}$, computed by means of Globular clusters (Bica et al. 2006). We obtained $d_{\odot}=$ $1.78 \pm 0.1 \mathrm{kpc}$ for the NGC 6357 complex based on the individual determinations for Pismis 24, BDS 101, ESO 392-SC 11, and VVV CL167 (Table 2).

\subsubsection{Pismis 24}

The decontaminated CMD of Pismis 24 (Fig. 3) presents a relatively vertically populous MS. It has a large population of red faint stars belonging to the PMS, making it a rather massive object in the sample. This cluster contains 12 known massive

\footnotetext{
6 http://stev.oapd.inaf.it/cgi-bin/cmd
}

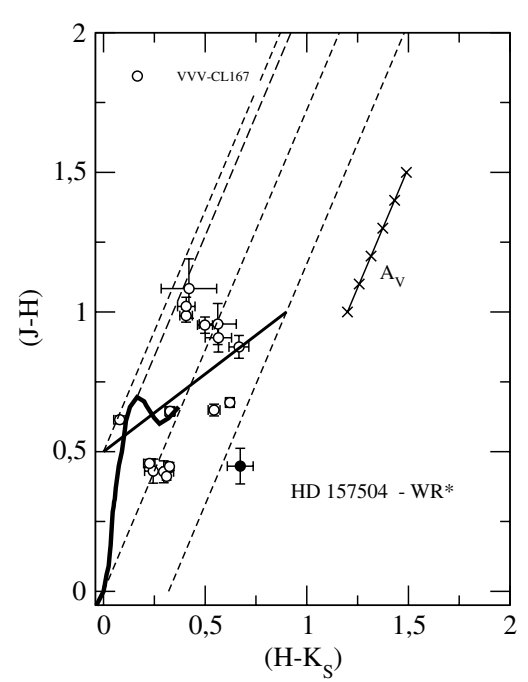

Fig. 5. Colour-colour diagram $\left((J-H) \times\left(H-K_{\mathrm{S}}\right)\right)$ of the decontaminated stars in VVV CL167. The continuous line represents the intrinsic distribution of spectral types and the continuous straight line indicates the undereddened locus of $\mathrm{T}$ Tauri stars. The redenning vector corresponds to $A_{V}=5$. Reddening directions for M 5 giants, O3, and T Tauri stars are shown as dashed lines.

stars, three of them (Pis24-1NE, Pis24-1SW, and Pismis 24-17) with $\sim 100 M_{\odot}$ each (Maíz Apellániz et al. 2007). These stars are absent from 2MASS and are saturated in VVV. Consequently, they do not appear in our CMDs. There is a debate in the literature on the distance of Pismis 24, and published estimates range from 1.0 to $3.0 \mathrm{kpc}$. We fitted a set of Padova isochrones $(0.2$, 1,3 , and $5 \mathrm{Myr}$ ) (Fig. 3), leading to $E\left(J-K_{\mathrm{S}}\right)=1.01 \pm 0.01$ $\left(E(B-V)=1.75 \pm 0.10\right.$ or $\left.A_{V}=5.87 \pm 0.06\right)$. The observed and absolute distance moduli are $(m-M)_{J}=13.20 \pm 0.10$ and $(m-M)_{\mathrm{O}}=11.50 \pm 0.10$, respectively, and $d_{\odot}=2.0 \pm 0.1 \mathrm{kpc}$. We estimated the mass of the PMS population of Pismis 24 by counting the number of PMS stars and multiplying it by a mean PMS stellar mass. We obtained $153 M_{\odot}$. For the mean PMS stellar mass, we assumed an initial mass function of Kroupa (2001) between 0.08 and $7 M_{\odot}$, which results in a mean mass of $0.6 M_{\odot}$ (Bonatto \& Bica 2010). For the MS stars in Fig. 3 the sum of the stellar masses is $80 M_{\odot}$. Finally, we added the three saturated supermassive stars not included in the VVV photometry, which makes the total $533 \pm 50 M_{\odot}$. We conclude that Pismis 24 is not an extremely massive cluster, but has an intermediate mass that is mostly stocked in its massive stars.

\subsubsection{BDS 101}

BDS 101 is a compact cluster. Differently from Pismis 24, its CMD (Fig. 3) shows a poorly populated MS and a rich PMS. The single star that occupies the MS is an $5^{7}$ star. We fitted a set of Padova isochrones (0.2, 1, 3, 5, and $7 \mathrm{Myr}$ ) (Fig. 3), leading to $E\left(J-K_{\mathrm{S}}\right)=1.13 \pm 0.01(E(B-V)=1.95 \pm 0.10$ or $\left.A_{V}=6.57 \pm 0.06\right)$. The observed and absolute distance moduli are $(m-M)_{J}=13.0 \pm 0.10$ and $(m-M)_{\mathrm{O}}=11.09 \pm 0.10$, respectively, and $d_{\odot}=1.66 \pm 0.09 \mathrm{kpc}$. The computed parameters are consistent with those of Pismis 24, indicating that BDS 101 is also embedded in the NGC 6357 complex. The total mass estimate for BDS 101 is $106 \pm 10 M_{\odot}$. Although a prominent cluster, it is not massive.

7 SIMBAD. 
Table 2. Derived fundamental parameters for the star clusters towards NGC 6357.

\begin{tabular}{lcccc}
\hline \hline Cluster & $\begin{array}{c}A_{V} \\
(\mathrm{mag}) \\
(2)\end{array}$ & $\begin{array}{c}\text { Age } \\
(\mathrm{Myr}) \\
(3)\end{array}$ & $\begin{array}{c}d_{\odot} \\
(\mathrm{kpc}) \\
(4)\end{array}$ & $\begin{array}{c}R_{\mathrm{GC}} \\
(\mathrm{kpc}) \\
(5)\end{array}$ \\
\hline Pismis 24 & $5.87 \pm 0.06$ & $5 \pm 2$ & $2.0 \pm 0.1$ & $5.3 \pm 0.1$ \\
BDS 101 & $6.57 \pm 0.06$ & $5 \pm 2$ & $1.7 \pm 0.1$ & $5.6 \pm 0.1$ \\
ESO 392-SC 11 & $6.69 \pm 0.06$ & $5 \pm 2$ & $1.9 \pm 0.1$ & $5.4 \pm 0.1$ \\
VVV CL164 & $15.9 \pm 0.09$ & $(5 \pm 2) \times 10^{3}$ & $1.4 \pm 0.1$ & $5.9 \pm 0.1$ \\
VVV CL167 & $4.94 \pm 0.06$ & $9 \pm 2$ & $1.6 \pm 0.1$ & $5.6 \pm 0.1$ \\
\hline
\end{tabular}

Notes. Column 2: $A_{V}$ absorption in the cluster central region. Column 3: age, from VVV photometry. Column 4: distance to the Sun. Column 5: $R_{\mathrm{GC}}$ distance of the object to the Galactic center.

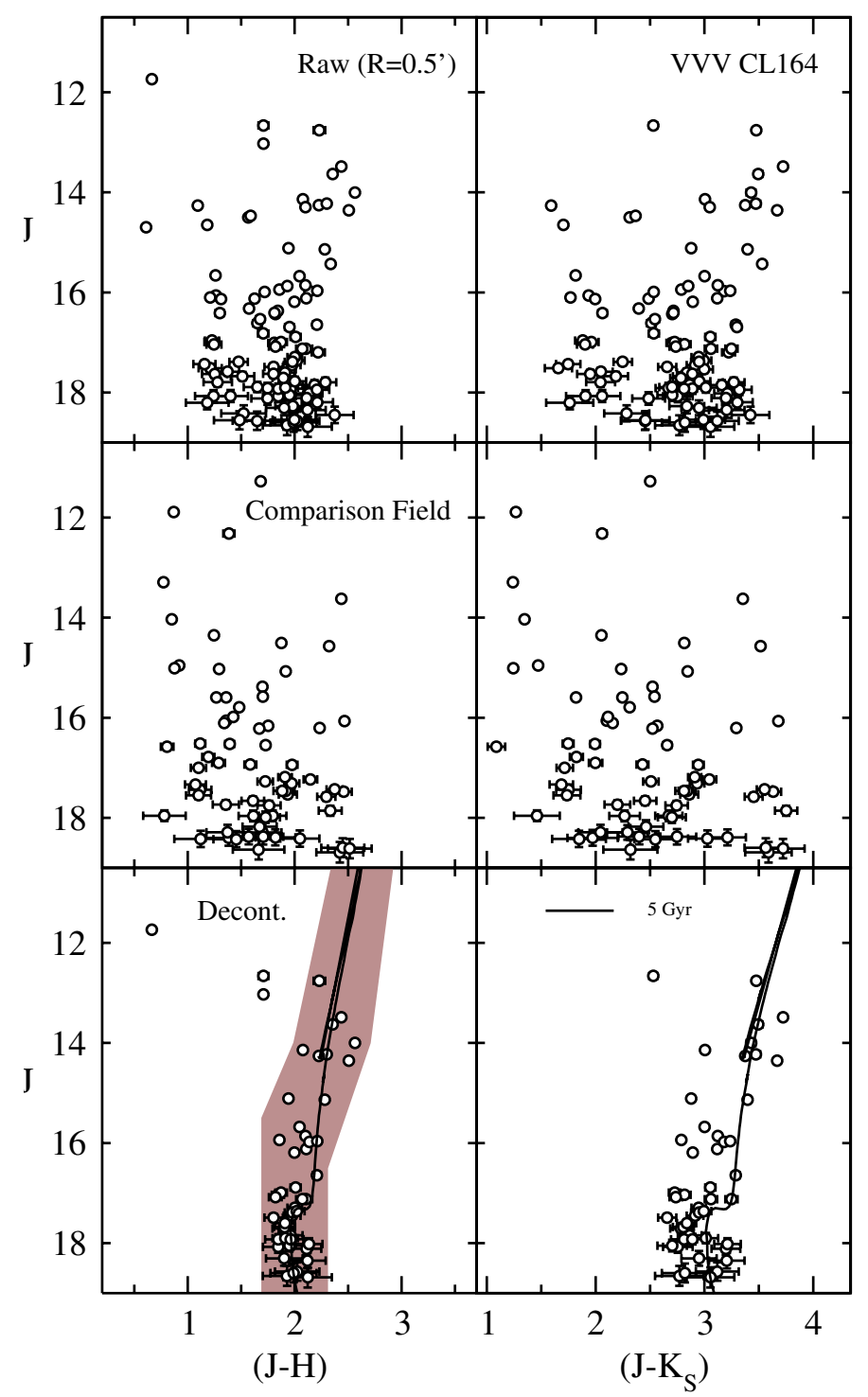

Fig. 6. Top-left panel: observed $J \times(J-H) \mathrm{CMD}$ of the region $R<$ 0.5 arcmin of VVVCL164. Middle-left panel: equal-area extraction from the comparison field. Bottom-left panel: the decontaminated CMD best-fit Padova isochrone (5 Gyr) together with the CM filter (brown polygon) used to isolate the MS and giants. Right panels: the same for $J \times\left(J-K_{\mathrm{S}}\right)$.

\subsubsection{VVV CL167}

This cluster contains the WR star WR 93 (van der Hucht 2001). Historically, this star has been considered a probable member of

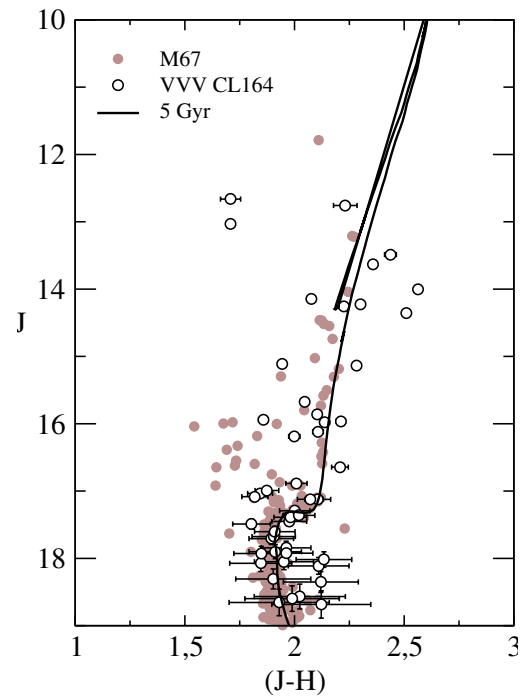

Fig. 7. Open cluster VVV CL164: comparison between the decontaminated $J \times(J-H)$ CMD for the region $R<0.5 \operatorname{arcmin}$ of VVV CL164 (empty circles) and the region $R<10$ acrmin of M 67 (filled circles). The best-fit to VVV CL164 is a Padova isochrone of 5 Gyr.

Pismis-24 (Massey et al. 2001). WR stars typically have masses in the range 10-25 $M_{\odot}$ and present strong, broad emission lines of He and $\mathrm{N}(\mathrm{WN})$ or He, C, and O (WC) (Meynet \& Maeder 2005). Recently, clusters containing WR stars were studied with the VVV (Chené et al. 2012). We point out that WR 93 is located too far $\left(\sim 5^{\prime}\right.$ west) from Pismis 24 . The image (Fig. 2) and the CMD (Fig. 4) indicate that a star cluster surrounds WR 93. We identify very few PMS stars in the CMD (Fig. 4). We adopted as best fit a Padova isochrone of 9 Myr (Fig. 4) that produces $E\left(J-K_{\mathrm{S}}\right)=0.85 \pm 0.01(E(B-V)=1.47 \pm 0.10$ or $\left.A_{V}=4.94 \pm 0.06\right)$. We derived the observed and absolute distance moduli as $(m-M)_{J}=12.50 \pm 0.10$ and $(m-M)_{\mathrm{O}}=11.07 \pm 0.10$, respectively. The distance to the Sun is $d_{\odot}=1.6 \pm 0.1 \mathrm{kpc}$. In the colour-colour diagram (Fig. 5) the star WR 93 shows $K_{\mathrm{S}}$ excess. A similar $K_{\mathrm{S}}$ excess was observed for massive stars in the $\sim 14$ Myr open cluster NGC 4755 (Bonatto et al. 2006a). The CMD of VVV CL-167 essentially contains only MS stars and WR 93. We obtain a cluster mass of $50 \pm 5 M_{\odot}$. The cluster age and mass point to a dynamically evolved cluster.

\subsubsection{VVV CL164 - a projected OC next to the complex}

The decontaminated CMDs $J \times(J-H)$ and $J \times\left(J-K_{\mathrm{S}}\right)$ are shown in the bottom panels of Fig. 6. We also include the CM filter on the $J \times(J-H)$ decontaminated CMD. The remaining stars populate sequences of typical intermediate-age 


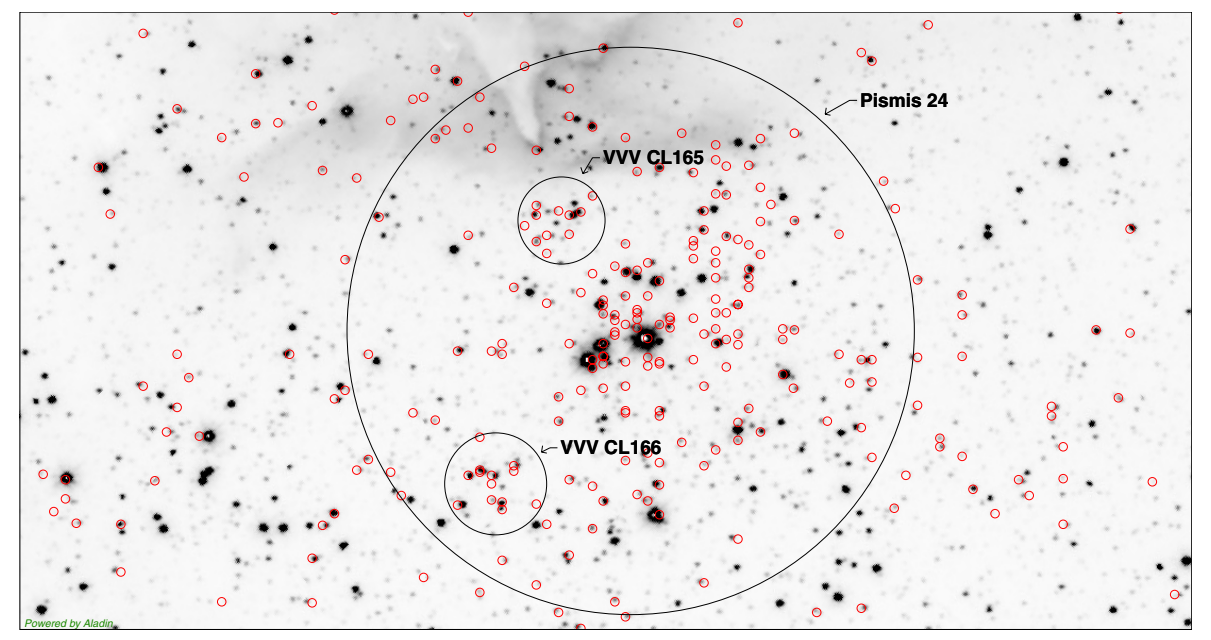

Fig. 8. $5^{\prime} \times 3^{\prime}$ VVV $K_{S}$ image of Pismis 24 area (large circle). The probable subclusters VVV CL165 (top circle) and VVV CL166 (bottom circle) are indicated. The small red circles indicate YSOs (Fang et al. 2012). The shift between the stars in the VVV image and the YSOs from the catalog of Fang (2MASS data) is a consequence of the difference of angular resolution of each survey, $0.34^{\prime \prime}$ and $2.0^{\prime \prime}$ respectively. The radius of the black circle is given in Table 1.
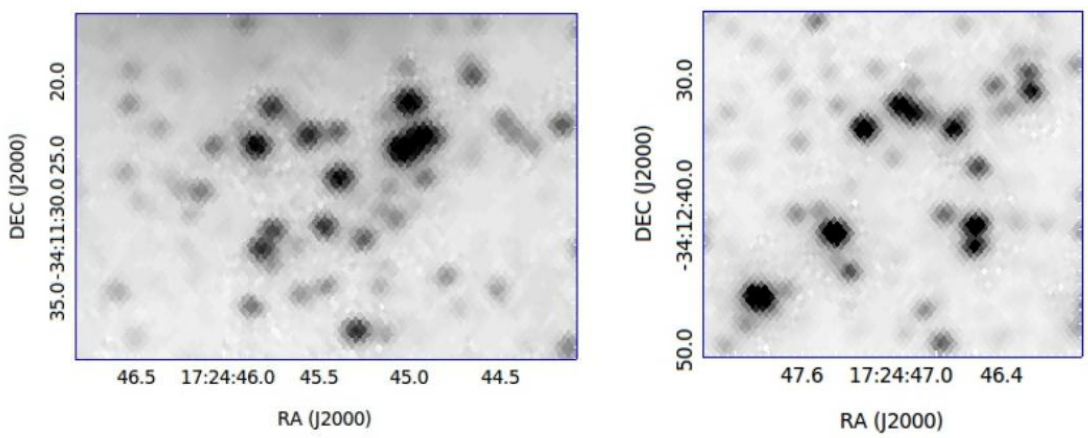

Fig. 9. $K_{\mathrm{S}}$ VVV zoomed images of the probable subclusters located in Pismis 24. Left panel: VVV CL165. Right panel: VVV CL166.

open clusters, with evidence of a red clump and a turn-off (TO) in both colours. Blue stragglers appear to be present as well. VVV CL164 presents relatively large errors at the TO level $(J \sim 18-19 \mathrm{mag}$ ). We matched a set of Padova isochrones (ages between 3 and $7 \mathrm{Gyr}$ ) and adopted $5 \mathrm{Gyr}$ as the best solution (Fig. 6). We obtain $E(J-H)=1.70 \pm 0.01 .(E(B-V)=4.73 \pm$ 0.15 or $\left.A_{V}=15.9 \pm 0.1\right)$. The $A_{V}$ value is considerably higher than for the other clusters. The observed and absolute distance moduli are $(m-M)_{J}=15.30 \pm 0.10$ and $(m-M)_{\mathrm{O}}=10.69 \pm 0.10$, respectively, resulting $d_{\odot}=1.37 \pm 0.07 \mathrm{kpc}$. We conclude that VVV CL164 is an intermediate-age cluster $(5 \pm 2 \mathrm{Gyr})$ that is projected next to the NGC 6357 complex.

Contrasting with the young clusters in the complex, the CMD of VVV CL164 presents MS, TO, and blue straggler stars, similar to those of M 67 built with 2MASS data (Fig. 7). We estimated the mass of this relatively old open cluster by adding stars throughout the decontaminated CMD using the stellar masses for M67 according to Bonatto \& Bica (2003). We obtained $\approx 200 M_{\odot}$ for VVV CL164.

\subsection{Additional case: subclustering in Pismis 24?}

The spatial distribution of YSOs in a cluster provides insights into the fragmentation processes that lead to the formation, of protostellar cores, evidence for triggered star formation and the subsequent dynamical evolution of the stars as they evolve from the protostellar phase to the MS (Megeath et al. 2004).
In Fig. 8 we show the YSOs from Fang et al. (2012) in the area of Pismis 24. Pismis 24 appears to have two close neighbours that are possible subclusters of YSOs. Zoomed VVV images of these objects suggest clusters (Fig. 9). The stellar density excesses in the RDP (Sect. 4.5) of Pismis 24 produced by these two possible subclusters are indicated in Fig. 12. The formation of these young stars may have been influenced by winds from the nearby OB stars in the core of Pismis 24. A possible fate of the ensemble is merging with the more massive cluster (McMillan et al. 2007; Pang et al. 2013).

\subsection{EC ESO 392-SC 11 (AHO3 J1725-34.4)}

Rahman et al. (2011a,b) have observed, in particular using GLIMPSE images and spectroscopy of luminous stars, far OB associations in the Galaxy which appear to be more massive than $10^{4} M_{\odot}$. In Fig. 10 we show a VVV $K_{\mathrm{s}}$ image of ESO 392-SC 11, where the compact cluster near the NW edge is BDS 101. The RDP of ESO 392-SC 11 appears to be rather flat (Fig. 13) like that of an association.

The decontaminated CMD $J \times\left(J-K_{\mathrm{S}}\right)$ is shown in the bottom panel of Fig. 11. We fitted a set of Padova isochrones $(0.2,1,3$ and $5 \mathrm{Myr})$, leading to $E\left(J-K_{\mathrm{S}}\right)=1.15 \pm 0.01(E(B-V)=1.99 \pm$ 0.10 or $\left.A_{V}=6.69 \pm 0.06\right)$. The observed and absolute distance moduli are $(m-M)_{J}=13.3 \pm 0.10$ and $(m-M)_{\mathrm{O}}=11.36 \pm$ 0.10 , respectively, resulting in $d_{\odot}=1.87 \pm 0.10 \mathrm{kpc}$. This set 


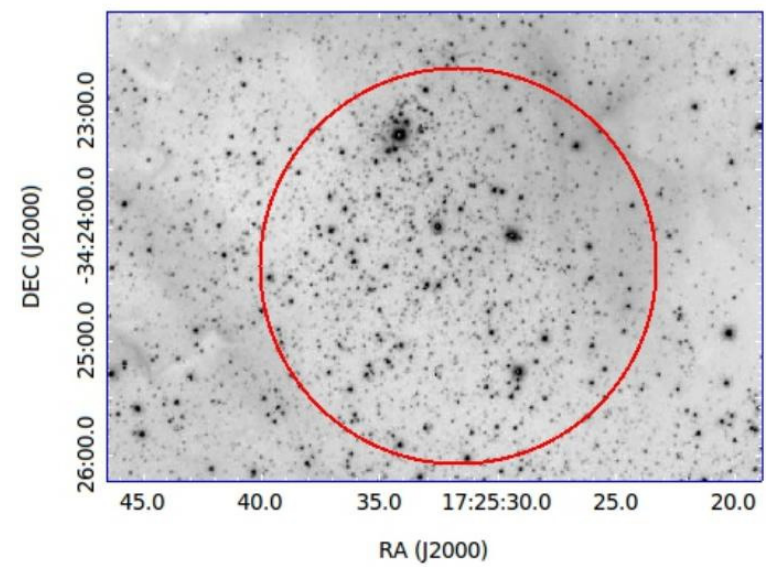

Fig. 10. $K_{\mathrm{S}} \mathrm{VVV}$ image of ESO392-SC11. The circle indicates the object area. The compact cluster near the northern edge is BDS 101.

of parameters shows that ESO 392-SC 11 is part of NGC 6357 complex, as are Pismis 24 and BDS 101.

The off-Galaxy position of the Magellanic Clouds enaldes size measurements of their stellar associations. Bica \& Schmitt (1995) and Bica et al. (1999) measured size and position angle for 3226 associations in the Magellanic system (LMC, SMC, and Bridge). In the LMC, Lucke \& Hodge (1970) reported diameters in the range 15-350 pc, while in the SMC, Hodge (1985) derived 20 to $140 \mathrm{pc}$. The smallest detected associations in the SMC (Bica \& Schmitt 1995) and LMC (Bica et al. 1999) have diameters of 5 and $7 \mathrm{pc}$, respectively, while the largest ones typically have $200 \mathrm{pc}$. The RDP radius of ESO 392-SC 11 is 3.3' (Table 3). This value corresponds to a diameter of $2.6 \mathrm{pc}$, which is lower than the lower limit of the associations in the Magellanic Clouds.

Some of the Magellanic Cloud associations were studied with HST, reaching the PMS population. LH 95 in the LMC has a diameter of about $40 \mathrm{pc}$ and includes a series of PMS clusters (Gouliermis et al. 2007). The total mass of LH 95 with HST is $3000 M_{\odot}$ (Da Rio et al. 2012). In diagnostic diagrams (Sect. 5) that involve structural parameters and mass ESO 392-SC 11 behaves like a star cluster, and not like an association. As a cautionary remark, the Magellanic Clouds and the Galaxy have different tidal environments that are expected to affect the fragmentation of the molecular clouds.

Since ESO392-SC11 consists of PMS stars and contains only a few MS stars, its association appearence will certainly change to that of a star cluster when more PMS stars evolve to the MS. Thus our results suggest that ESO392-SC11 is in a preassociation stage. If the region loses gas and dust, then the system may be destabilized and later evolve dynamically to an association, after a star-cluster phase.

\subsection{Stellar density profiles}

We used projected stellar RDPs, defined as the stellar number density around the cluster center, to derive structural parameters. Noise in the RDPs was minimised with CM filters, which exclude stars with colours that are incompatible with those of the cluster. Our group has shown that this filtering procedure considerably enhances the RDP contrast relative to the comparison field, especially in crowded fields (Bonatto \& Bica 2007a). The CM-filtered RDPs of the clusters are shown in Fig. 12.

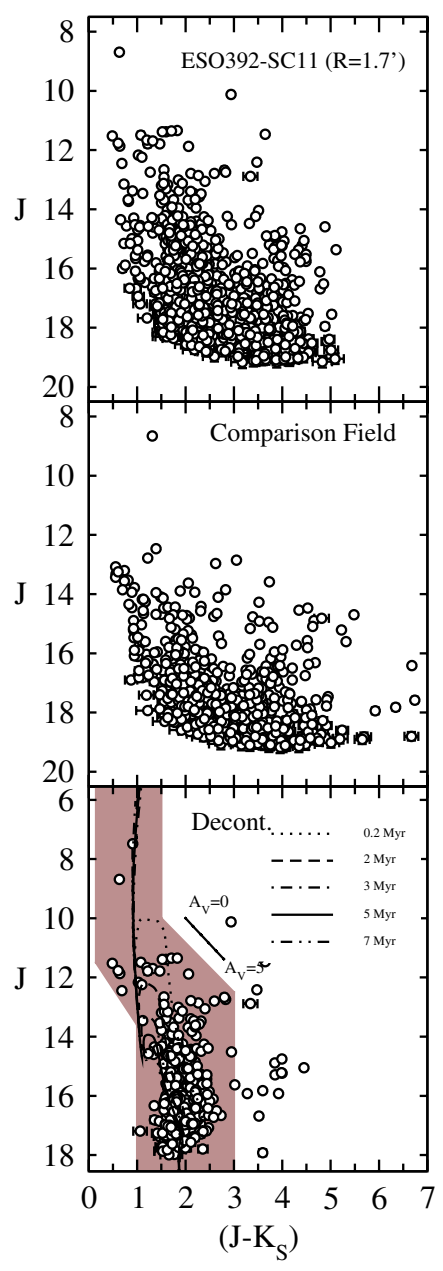

Fig. 11. Top panel: observed $J \times\left(J-K_{\mathrm{S}}\right) \mathrm{CMD}$ of the region $R<$ 1.7 arcmin of ESO 392-SC 11. Middle panel: equal-area extraction from the comparison fields. Bottom panel: the decontaminated CMD together with the CM filter (brown polygon). MS and PMS isochrones are given.

Whenever possible, we fitted a two-parameter King-like profile $\sigma(R)=\sigma_{\mathrm{bg}}+\sigma_{0} /\left(1+\left(R / R_{\mathrm{c}}\right)^{2}\right)($ King 1966) adapted to star counts, where $\sigma_{0}$ and $\sigma_{\mathrm{bg}}$ are the central and residual stellar densities, and $R_{\mathrm{c}}$ is the core radius. The structural $\sigma_{0}$ and $R_{\mathrm{c}}$ parameters were derived from the fit, while $\sigma_{\mathrm{bg}}$ was previously measured in the comparison field and kept constant. The extent of the clusters $\left(r_{\mathrm{RDP}}\right)$ is defined as the radius where the RDP meets the field-star count level. The best-fit solutions and other results are listed in Table 3. We point out that we are dealing with very young clusters (except VVV CL164). Thus we do not expect relaxed systems, but interestingly, the profiles nevertheless describe the cluster RDP, such as the decontaminated profile of Pismis 24.

The absence of a central peak in the RDP of Pismis 24 (Fig. 12) is probably a consequence of the overshadowing produced by the bright stars on the surrounding faint members. The RDP appears to present two excesses, supporting the existence of subclusters there (VVV CL165 and VVV CL166).

The irregular RDP of VVV CL167 could not be fitted by a King-like profile (Fig. 12). This suggests that VVV CL167 is a dynamically evolved cluster as a consequence of general mass loss, in particular winds from WR 93 and other less massive stars that remove the interstellar material in the cluster (Tutukov 1978; Goodwin \& Bastian 2006). 
E. F. Lima et al.: Star clusters in NGC 6357
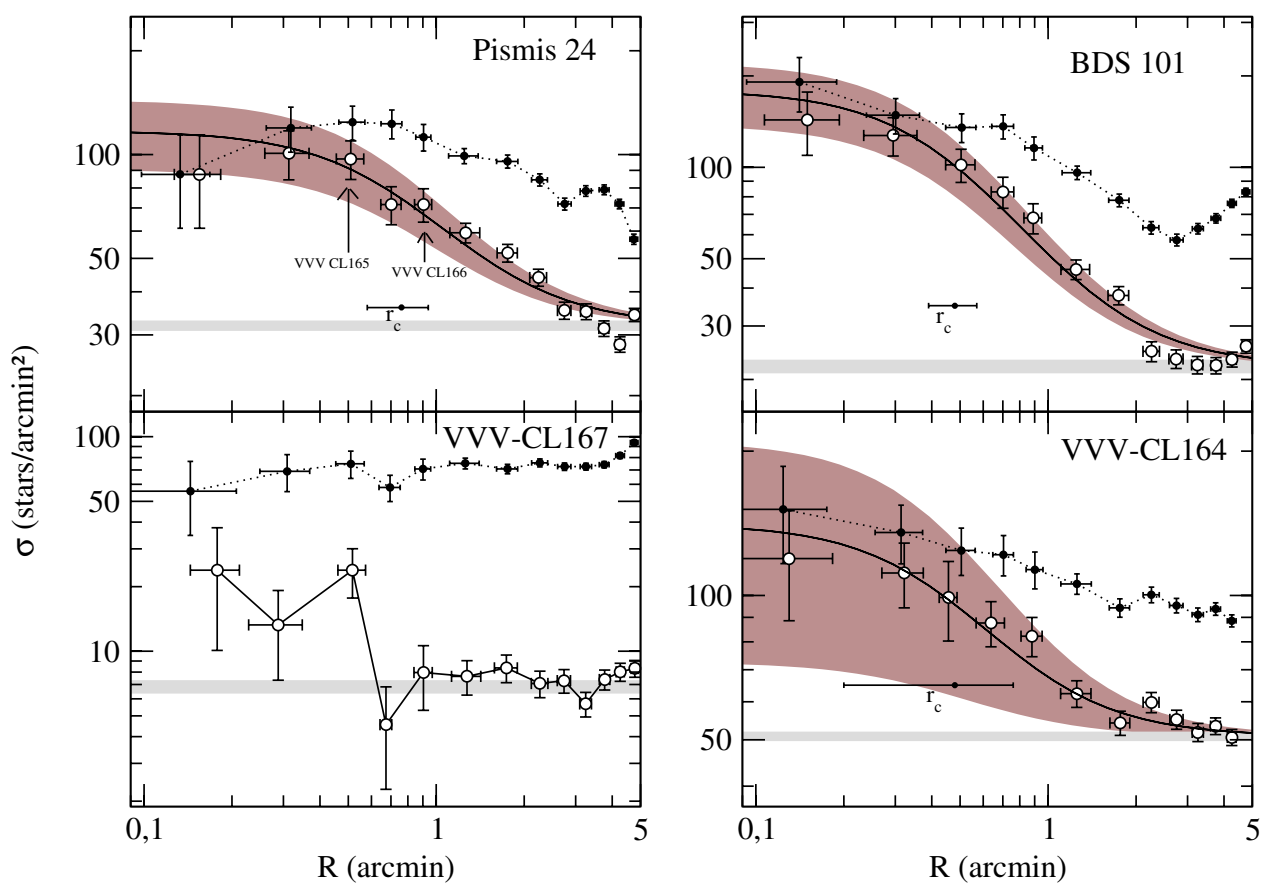

Fig. 12. Colour-magnitude filtered RDPs (empty circles). The dots represent the raw cluster profile. The empty circles show the cleaned profiles. The best King-like fit is shown, when possible. The $1 \sigma$ fit uncertainty is represented by the dark-shaded strip along the fit. The background level is the light-shaded region. The core radius $\left(r_{\mathrm{c}}\right)$ is indicated.

Table 3. Cluster structural parameters.

\begin{tabular}{ccccccc}
\hline \hline Cluster & $\begin{array}{c}\sigma_{0} \\
\left(\mathrm{stars} /{ }^{2}\right)\end{array}$ & $\begin{array}{c}r_{\mathrm{c}} \\
\left({ }^{\prime}\right)\end{array}$ & $\begin{array}{c}R_{\mathrm{c}} \\
(\mathrm{pc})\end{array}$ & $\begin{array}{c}\sigma_{\mathrm{bg}} \\
\left(\mathrm{stars} /{ }^{2}\right)\end{array}$ & $\begin{array}{c}r_{\mathrm{RDP}} \\
\left({ }^{\prime}\right)\end{array}$ & $\begin{array}{c}R_{\mathrm{RDP}} \\
(\mathrm{pc})\end{array}$ \\
\hline Pismis 24 & $85 \pm 26$ & $0.76 \pm 0.20$ & $0.44 \pm 0.11$ & $31.9 \pm 0.9$ & $3.2 \pm 0.3$ & $1.9 \pm 0.2$ \\
BDS 101 & $157 \pm 40$ & $0.48 \pm 0.21$ & $0.23 \pm 0.04$ & $22.1 \pm 1.0$ & $2.3 \pm 0.2$ & $1.1 \pm 0.1$ \\
ESO392-SC 11 & $104 \pm 29$ & - & - & $36 \pm 1.1$ & $3.3 \pm 0.3$ & $1.8 \pm 0.2$ \\
VVV CL167 & $24 \pm 14$ & - & - & $6.8 \pm 0.4$ & $2.3 \pm 0.2$ & $1.1 \pm 0.1$ \\
VVV CL164 & $90 \pm 68$ & $0.48 \pm 0.28$ & $0.19 \pm 0.10$ & $50.8 \pm 1.0$ & $3.3 \pm 0.3$ & $1.3 \pm 0.1$ \\
\hline
\end{tabular}

Notes. Column 1: cluster designation. Column 2: central density. Column 3: core radius (arcmin). Column 4: core radius (pc), assuming distances to the Sun in Table 2. Column 5: background density. Column 6: cluster RDP radius (arcmin). Column 7: cluster RDP radius (pc).

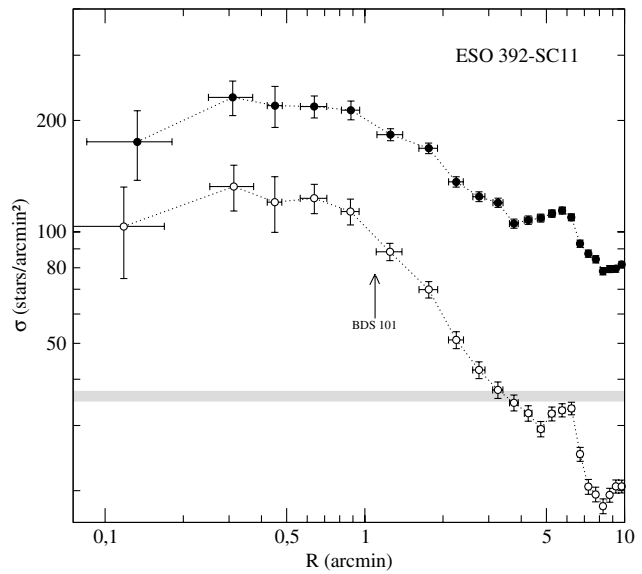

Fig. 13. Same as Fig. 12, for ESO 392-SC 11. The location of BDS 101 in the profile is indicated.

ESO 392-SC 11 has a peculiar profile (Fig. 13). We could not fit any King-profile. The superimposed contribution of the compact cluster BDS 101 could not be subtracted.

\section{Object structure}

We used the structural parameters from Sect. 4.5 to investigate possible cluster evolutionary effects (Gieles \& Portegies Zwart 2011; Saurin et al. 2012). Diagnostic diagrams for the dynamical evolution of star clusters have been built by our group in a series of papers (e.g. Bonatto \& Bica 2009b, 2010; Bica et al. 2008).

In Fig. 14 we show the estimated stellar mass compared with the extent RDP radius. For comparison purposes, we include a sample of nearby young clusters studied by our group following similar methods. The clusters are NGC 2244 and NGC 2239 (Bonatto \& Bica 2009b), NGC 6611 (Bonatto et al. 2006b), NGC 6823 (Bica et al. 2008), vdB 92 (Bonatto \& Bica 2010), Pismis 5, vdB 80, NGC 1931, and BDSB 96 (Bonatto \& Bica $2009 \mathrm{c})$. We also include the large and populous associations Collinder 197 (Bonatto \& Bica 2010), Bochum 1 (Bica et al. 2008), and Trumpler 37 (Saurin et al. 2012). In addition, we show LH 95, a massive LMC association studied with HST (Da Rio et al. 2012). The general behaviour of the clusters and associations appears to present a flattening effect, in the sense that associations tend to inflate while keeping large masses. The clusters in NGC 6357 present low-to-intermediate integrated 


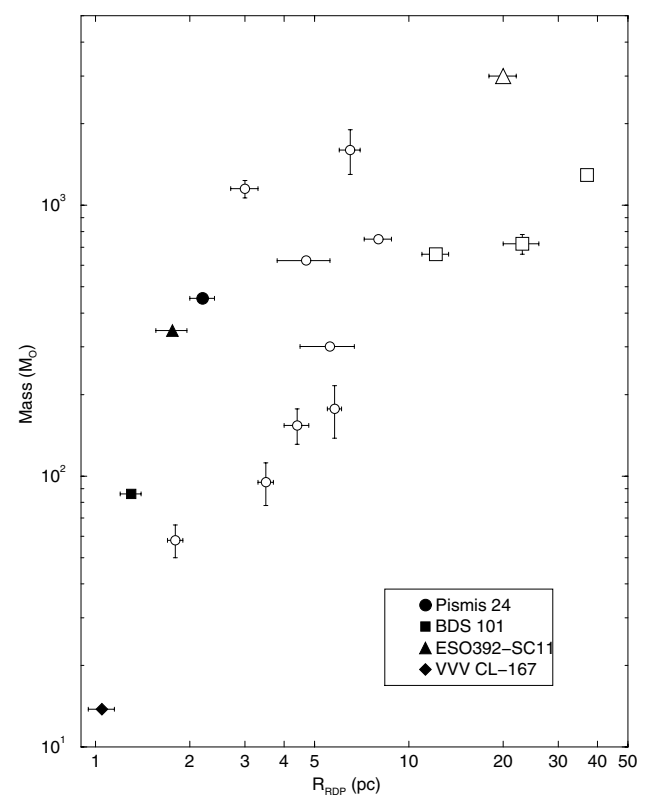

Fig. 14. Cluster or association mass $\times$ extent RDP radius. Open circles: comparison cluster sample; open squares: associations or evolving associations; open triangle: the LMC association LH 95. The present sample is indicated.

stellar masses, but they are systematically smaller than the comparison clusters. Since they are located in the Sagittarius Arm, they are possibily affected by tidal effects, even at the phase of cloud fragmentation.

Another way of investigating this issue is by comparing the cluster radius $\left(R_{\mathrm{RDP}}\right)$ with the half-starcount radius $\left(R_{\mathrm{hSC}}\right)$. The latter is equivalent to the half-light radius commonly used in star cluster studies in the outer parts of the Galaxy (e.g. Belokurov et al. 2009). The results are shown in Fig. 15, where again we use the same comparison clusters and associations (except LH 95) as in Fig. 14. The comparison clusters follow a powerlaw scaling relation for $R_{\mathrm{RDP}}$ and $R_{\mathrm{hSC}}$. Again, the associations Collinder 197, Bochum 1 and Trumpler 37 appear to be inflated at the outskirts compared with the cluster-scaling relation. The clusters in NGC 6357 are quite small compared with the young reference clusters (Fig. 14). Note that ESO 392-SC11 (Fig. 13) lies in a similar locus as Pismis 24 (Fig. 14), thus implying a cluster behaviour.

\section{Discussion and conclusions}

We studied the NGC 6357 star-forming complex with VVV photometry. The main focus of our study were seven stellar clusters in the area. Pismis 24 is a relatively populous EC, with a developed MS and PMS. BDS 101 is compact, with a CMD dominated by PMS stars. The EC VVV CL167 includes a WR star and is significantly older than Pismis 24 and BDS 101. VVV CL165 and VVV CL166 appear to be subclusters related to Pismis 24. ESO392-SC11 is an interesting object, probably in a pre-association stage.

VVV CL164 to VVV CL167 were discovered in the present paper. From the clusters belonging to the complex (Pismis 24, BDS 101, ESO 392-SC 11, and VVVCL167), we derived a mean distance of $d_{\odot}=1.78 \pm 0.1 \mathrm{kpc}$ to NGC 6357. We also detected an open cluster of intermediate age (VVV CL164) projected next to the complex. Evidence was found that these clusters in NGC 6357 are smaller than nearby embedded clusters.

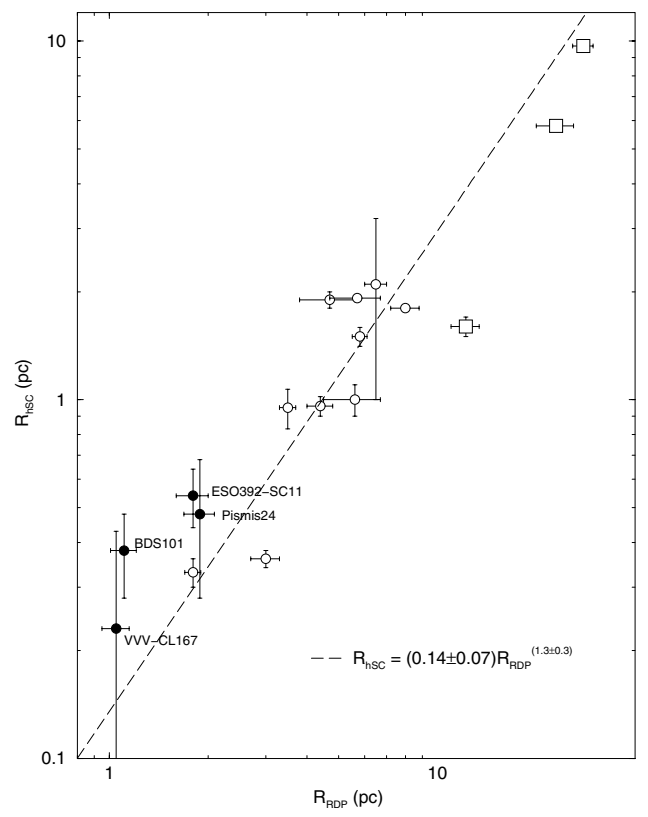

Fig. 15. Distribution of the half-starcount radius $x$ extent radius. Symbols as in Fig. 14.

The NGC 6357 complex, now with several analysed ECs, resembles more its neighbouring complex NGC 6334 (Feigelson et al. 2009; Russeil et al. 2012, 2013).

In the cluster mass calculations (Sect. 4.2) we considered stars in the decontaminated area (corresponding to the radii given in Col. 6 of Table 1). Pismis 24 and ESO 392-SC 11 have intermediate masses, while the other clusters in NGC 6357 have low masses. VVV CL167 probably is unstable and will lose additional gas mass in the coming Myr (Bastian \& Goodwin 2006).

In some aspects, the NGC 6357 complex emulates a starforming dwarf galaxy, in the sense that in addition to a rich content of interstellar features, it hosts embedded clusters, which indicates at least two stellar generation events.

Acknowledgements. We thank the referee for important comments and suggestions. We acknowledge support from the Brazilian Institution CNPq. We gratefully acknowledge use of data from the ESO Public Survey programme ID 179.B-2002 taken with the VISTA telescope, data products from the Cambridge Astronomical Survey Unit. R.K.S. acknowledges support from CNPq/Brazil through projects 310636/2013-2 and 481468/2013-7. We acknowledge use of the VizieR Catalogue Service operated at the CDS, Strasbourg, France. This publication makes use of data products from the Two Micron All Sky Survey, which is a joint project of the University of Massachusetts and the Infrared Processing and Analysis Centre/California Institute of Technology, funded by the National Aeronautics and Space Administration and the National Science Foundation.

\section{References}

Archinal, B. A., \& Hynes, S. J. 2003, Star clusters (Willmann-Bell) Bastian, N., \& Goodwin, S. P. 2006, MNRAS, 369, L9

Belokurov, V., Walker, M. G., Evans, N. W., et al. 2009, MNRAS, 397, 1748

Bica, E., Dutra, C. M., Soares, J., \& Barbuy, B. 2003, A\&A, 404, 223

Bica, E., Bonatto, C., Barbuy, B., \& Ortolani, S. 2006, A\&A, 450, 105

Bica, E., Bonatto, C., \& Dutra, C. M. 2008, A\&A, 489, 1129

Bica, E. L. D., \& Schmitt, H. R. 1995, ApJS, 101, 41

Bica, E. L. D., Schmitt, H. R., Dutra, C. M., \& Oliveira, H. L. 1999, AJ, 117, 238

Bohigas, J., Tapia, M., Roth, M., \& Ruiz, M. T. 2004, AJ, 127, 2826

Bonatto, C., \& Bica, E. 2003, A\&A, 405, 525

Bonatto, C., \& Bica, E. 2007a, A\&A, 473, 445

Bonatto, C., \& Bica, E. 2007b, MNRAS, 377, 1301

Bonatto, C., \& Bica, E. 2009a, MNRAS, 392, 483

Bonatto, C., \& Bica, E. 2009b, MNRAS, 394, 2127 
Bonatto, C., \& Bica, E. 2009c, MNRAS, 397, 1915

Bonatto, C., \& Bica, E. 2010, A\&A, 516, A81

Bonatto, C., Bica, E., Ortolani, S., \& Barbuy, B. 2006a, A\&A, 453, 121

Bonatto, C., Santos, Jr., J. F. C., \& Bica, E. 2006b, A\&A, 445, 567

Bonatto, C., Bica, E., \& Lima, E. F. 2012a, MNRAS, 420, 352

Bonatto, C., Lima, E. F., \& Bica, E. 2012b, A\&A, 540, A137

Borissova, J., Chene, A. N., Ramirez Alegrìa, S., et al. 2014, A\&A, in press, DOI 10.1051/0004-6361/201322483

Bressan, A., Marigo, P., Girardi, L., et al. 2012, MNRAS, 427, 127

Camargo, D., Bonatto, C., \& Bica, E. 2011, MNRAS, 416, 1522

Cappa, C. E., Barbá, R., Duronea, N. U., et al. 2011, MNRAS, 415, 2844

Carvalho, L., Saurin, T. A., Bica, E., Bonatto, C., \& Schmidt, A. A. 2008, A\&A, 485, 71

Chené, A.-N., Borissova, J., Clarke, J. R. A., et al. 2012, A\&A, 545, A54

Conti, P. S., \& Vacca, W. D. 1990, AJ, 100, 431

Da Rio, N., Gouliermis, D. A., Rochau, B., et al. 2012, MNRAS, 422, 3356

Damke, G., Barbá, R., \& Morrell, N. I. 2006, Rev. Mex. Astron Astrofis., 26, 180

Dias, W. S., Alessi, B. S., Moitinho, A., \& Lépine, J. R. D. 2002, A\&A, 389, 871

Fang, M., van Boekel, R., King, R. R., et al. 2012, A\&A, 539, A119

Feigelson, E. D., Martin, A. L., McNeill, C. J., Broos, P. S., \& Garmire, G. P. 2009, AJ, 138, 227

Felli, M., Persi, P., Roth, M., et al. 1990, A\&A, 232, 477

Friel, E. D. 1995, ARA\&A, 33, 381

Gieles, M., \& Portegies Zwart, S. F. 2011, MNRAS, 410, L6

Goodwin, S. P., \& Bastian, N. 2006, MNRAS, 373, 752

Gouliermis, D. A., Henning, T., Brandner, W., et al. 2007, ApJ, 665, L27

Hodge, P. 1985, PASP, 97, 530

Irwin, M. J., Lewis, J., Hodgkin, S., et al. 2004, in Optimizing Scientific Return for Astronomy through Information Technologies, eds. P. J. Quinn,

\& A. Bridger, SPIE Conf. Ser., 5493, 411

King, I. R. 1966, AJ, 71, 64

Kroupa, P. 2001, MNRAS, 322, 231
Lada, C. J., \& Lada, E. A. 2003, ARA\&A, 41, 57

Lauberts, A. 1982, ESO/Uppsala survey of the ESO(B) atlas (ESO)

Leisawitz, D., Bash, F. N., \& Thaddeus, P. 1989, ApJS, 70, 731

Lortet, M. C., Testor, G., \& Niemela, V. 1984, A\&A, 140, 24

Lucke, P. B., \& Hodge, P. W. 1970, AJ, 75, 171

Maíz Apellániz, J., Walborn, N. R., Morrell, N. I., Niemela, V. S., \& Nelan, E. P. 2007, ApJ, 660, 1480

Massey, P., DeGioia-Eastwood, K., \& Waterhouse, E. 2001, AJ, 121, 1050

McMillan, S. L. W., Vesperini, E., \& Portegies Zwart, S. F. 2007, ApJ, 655, L45

Megeath, S. T., Allen, L. E., Gutermuth, R. A., et al. 2004, ApJS, 154, 367 Meynet, G., \& Maeder, A. 2005, A\&A, 429, 581

Minniti, D., Lucas, P. W., Emerson, J. P., et al. 2010, New Astron., 15, 433

Moffat, A. F. J., \& Vogt, N. 1973, A\&AS, 10, 135

Moisés, A. P., Damineli, A., Figuerêdo, E., et al. 2011, MNRAS, 411, 705

Naylor, T., \& Jeffries, R. D. 2006, MNRAS, 373, 1251

Neckel, T. 1978, A\&A, 69, 51

Neckel, T. 1984, A\&A, 137, 58

Pang, X., Grebel, E. K., Allison, R. J., et al. 2013, ApJ, 764, 73

Persi, P., Ferrari-Toniolo, M., Roth, M., \& Tapia, M. 1986, A\&A, 170, 97

Rahman, M., Matzner, C., \& Moon, D.-S. 2011a, ApJ, 728, L37

Rahman, M., Moon, D.-S., \& Matzner, C. D. 2011b, ApJ, 743, L28

Russeil, D., Zavagno, A., Motte, F., et al. 2010, A\&A, 515, A55

Russeil, D., Zavagno, A., Adami, C., et al. 2012, A\&A, 538, A142

Russeil, D., Schneider, N., Anderson, L. D., et al. 2013, A\&A, 554, A42

Saito, R. K., Hempel, M., Minniti, D., et al. 2012, A\&A, 537, A107

Saurin, T. A., Bica, E., \& Bonatto, C. 2010, MNRAS, 407, 133

Saurin, T. A., Bica, E., \& Bonatto, C. 2012, MNRAS, 421, 3206

Skrutskie, M. F., Cutri, R. M., Stiening, R., et al. 2006, AJ, 131, 1163

Tutukov, A. V. 1978, A\&A, 70, 57

van der Hucht, K. A. 2001, New Astron. Rev., 45, 135

Walborn, N. R., Howarth, I. D., Lennon, D. J., et al. 2002, AJ, 123, 2754

Wang, J., Townsley, L. K., Feigelson, E. D., et al. 2007, ApJS, 168, 100

Wilson, T. L., Mezger, P. G., Gardner, F. F., \& Milne, D. K. 1970, A\&A, 6, 364 